\title{
La refundación de la Universidad de Guadalajara en 1925. La mística de la revolución inhibe su autonomía*
}

\author{
The refoundation of the University of Guadalajara in 1925. The mystique of \\ the revolution inhibits its autonomy
}

\author{
A refundação da Universidade de Guadalajara em 1925. A mística da \\ revolução inibe sua autonomia
}

\author{
Armando Martínez Moya ${ }^{1}$ \\ Universidad de Guadalajara (México) \\ Grupo de investigación HISULA - UPTC
}

Recepción: 20/05/2017

Evaluación: 07/11/2017

Aceptación: 05/01/2018

Artículo de investigación - Reflexión

DOI: https://doi.org/10.19053/01227238.8014

\section{RESUMEN}

Don José Guadalupe Zuno, Gobernador del Estado de Jalisco a partir de 1923, volvió a darle a la educación superior su carácter universitario, pues desde el cierre de la antigua universidad decimonónica y del Instituto de Ciencias del Estado en 1865, las escuelas superiores que funcionaron dependían directamente del gobierno del Estado. Al fundarse en 1925 la Universidad de Guadalajara el hecho representó un gran acontecimiento para el país, especialmente para la región noroccidente del país, fue la segunda universidad fundada y si bien la filosofía que fundamentaba su existencia se sustentaba en reivindicar su carácter laico, popular, científico y alejada de cualquier influencia religiosa, no contenía en su nombre ni en su normativa ni en su funcionamiento el título de Autónoma, no obstante que la influencia de la lucha y conquista de la autonomía cordobesa de 1918 era conocida en todo el continente y se desplegaba por el mundo -al grado que pocos años después, en 1829, la Universidad de México la conquistó también-. En realidad, en la Universidad de Guadalajara, este importante antecedente autonomista no se tomó en cuenta. La influencia de la revolución mexicana, cuyo periodo armado había recién concluido (1910-1917), mantenía como uno de sus principales reivindicaciones promover la educación y la cultura populares al pueblo. En este contexto Zuno materializaba esa gran aspiración popular fundando la universidad dentro de ese paradigma revolucionario, pero lo hacía bajo una óptica unipersonal,

\footnotetext{
* Resultado del proyecto de investigación "La Universidad Pedagógica y Tecnológica de Colombia en los desafíos del siglo XXI. Una prospectiva desde los estudiantes y docentes". Código: SGI 1965 financiado por la vicerrectoría de Investigaciones - UPTC y desarrollado por el Grupo de Investigación "Historia y Prospectiva de la Universidad Latinoamericana". HISULA.

1 Doctor en Historia, actualmente profesor de la Universidad de Guadalajara - México, es integrante del grupo de investigación, Historia y prospectiva de la Universidad Latinoamericana - HISULA de la UPTC clasificado en COLCIECIAS CATEGORIA A. Correo electrónico: mmarmando@gmail.com
} 
donde el gobierno del Estado y el rector fueron quienes asumieron la dirección universitaria, estableciendo un modelo directivista -puesto que los directores coparon el Consejo Universitario, y junto a ello desconocieron las representaciones estudiantiles, la elección democrática de profesores y sujetaron la universidad al régimen, es decir al gobierno en turno. El pretexto fue vincular el modelo con las necesidades y reclamos de una sociedad que se sacrificó por la revolución, pero mantenida como un ariete del Estado.

Palabras clave: José Guadalupe Zuno; Autonomía; representaciones estudiantiles; revolución mexicana; educación popular; caudillismo.

ABSTRACT
Don José Guadalupe Zuno, Governor
of the State of Jalisco since 1923, gave hi-
gher education its university character
again, because since the closing of the
old nineteenth-century university and
the Institute of State Sciences in 1865, the
higher schools directly depended of the
State. The foundation of the University of
Guadalajara in 1925 was a great event for
the country, especially for the northwes-
tern region. This was the second universi-
ty founded and although the philosophy
that founded its existence was based on
claiming its secular, popular, scientific
character and away from any religious in-
fluence, it did not contain in its name nor
in its regulations nor its operation the title
of Autonomous, despite the fact that the
influence of the struggle and conquest of
Cordovan autonomy in 1918 was known
throughout the continent and spread
throughout the world - to the extent that
a few years later, in 1829, the University
of Mexico also conquered it. In fact, at the
University of Guadalajara, this important
autonomist antecedent was not taken into
account. The influence of the Mexican Re-
volution, whose armed period had just
ended (1910-1917), kept as one of its main

claims to promote education and popular culture to the people.

In this context, Zuno materialized that great popular aspiration founding the university within that revolutionary paradigm, but he did it from a unipersonal perspective, where the government of the State and the rector were the ones who assumed the university direction, establishing in this way, a directivist model - since the directors took over the University Council, and additionaly they did not recognize the student representations, the democratic election of professors and they subjected the university to the regime, that is to say to the government in turn. The pretext was to link the model with the needs and claims of a society that sacrificed itself for the revolution, but that was assumed as a state battering ram.

Keywords: Jose Guadalupe Zuno; Autonomy; student representations; Mexican Revolution; popular education; warlordism.

\section{RESUMO}

Don José Guadalupe Zuno, governador do Estado de Jalisco desde 1923, voltou a conferir à educação superior seu caráter universitário, pois desde o encerramento da antiga universidade do século XIX e do Instituto de Ciências do Estado, em 1865, as escolas superiores que funcionavam dependiam diretamente do governo do Estado. Ao fundar-se a Universidade de Guadalajara em 1925, tal fato representou um grande acontecimento para o país, especialmente para a região norte ocidental do país. Foi a segunda universidade fundada, e a filosofia que fundamentava sua existência se sustentava em reivindicar seu caráter laico, popular, científico e livre de qualquer influência religiosa, não continha em seu nome, nem em sua normativa e nem em seu funcionamento o título de Autônoma, não obstante a influência da luta e conquista da autonomia de Córdoba de 1918 fosse conhecida em todo o continente e se espalhava pelo mundo - posto que pouco 
tempo depois, em 1892, a Universidade do México também conquistou. Na realidade, na Universidade de Guadalajara, este importante antecedente autonomista não foi levado em conta. A influência da revolução mexicana, cujo período armado havia sido recentemente concluído (1910-1917), mantinha como um de suas principais reivindicações a promoção da educação e da cultura populares ao povo. Neste contexto, Zuno materializava essa grande aspiração popular fundando a universidade dentro desse paradigma revolucionário, porém o fazia sob uma visão unipessoal, donde o governo do Estado e o reitor foram aqueles a assumir a direção universitária, estabelecendo um modelo diretivo - posto que os diretores assumiram o Conselho Universitário, e junto a ele desconheciam as representações estudantis, a eleição democrática de professores e sujeitando a universidade ao regime, isto é, ao governo em curso. O pretexto foi vincular o modelo às necessidades $\mathrm{e}$ demandas de uma sociedade que se sacrificou pela revolução, porém permaneceu como aríete do Estado.

Palavras-chave: Jose Guadalupe Zuno; Autonomia; representações estudiantis; revolução Mexicana; educação popular; caudillismo.

\section{INTRODUCCIÓN}

La relación entre refundación y autonomía ha sido poco estudiada en la Universidad de Guadalajara (UdG) debido al carácter unipersonal que significó su segundo establecimiento en 1925, puesto que fue un proyecto de un grupo político cultural que en una determinada cobertura política logró establecerla como una decisión de Estado. De ahí que buscar la influencia de Córdoba o de otros contextos para explicar su modelo al plantearse su establecimiento no ha sido hasta ahora un recurso muy utilizado por lo poco factible que parece resultar. Más bien se ha reflexionado desde las motivaciones y circunstancias internas y domésticas que dieron lugar a su cristalización. ${ }^{2} \mathrm{~A}$ diferencia de la Universidad de México, cuya característica más emblemática fue la conquista de su autonomía en $1929,{ }^{3}$ la de Guadalajara se significó particularmente por representar la consumación de un proyecto educativo del naciente Estado revolucionario (1910-1917-1925). Conforme a lo anterior, en este trabajo se buscarán desde lo documental e historiográfico, las fuentes de la época elementos que nos permitan identificar más diáfanamente su modelo y tratar con ello de descubrir algún vínculo -o en su caso las explicaciones de su ausencia respecto al gran acontecimiento de Córdoba-.

Conforme a lo anterior, y a partir de esa hipótesis, se plantean los antecedentes de lo que había significado históricamente la Universidad; las condiciones

2 Francisco Ayon Zester, Universidad de Guadalajara, (Guadalajara: Instituto Jalisciense de Antropología e Historia, 1986). Misael Gradilla Gramy, "El juego del poder y del saber" (Tesis doctoral. El Colegio de México. México, 1993). Alfredo Mendoza Cornejo, Historia de la Universidad de Guadalajara. 1696-1994, Vol. 2 (Guadalajara: Edición del autor, 2005). Marina Mantilla Trolle y Armando Martínez Moya, "La Universidad de Guadalajara a la hora de su fundación”) en Universidad de Guadalajara: más de dos siglos de historia, eds. Gloria Angélica Hernández Obledo (Guadalajara: Universidad de Guadalajara, 2013).

3 Renate Marsiske, "La autonomía universitaria. Una visión histórica y latinoamericana”, Perfiles Educativos XXXII, (2010): 9-26. Justo Cuño Bonito. "La universidad Latinoamericana en la encrucijada: amenazas, desafíos y soluciones". Revista Historia de la Educación Latinoamericana. Vol. 18 No. 26 (2016): 241- 277. 
políticas y el contexto que gravitaba. Para ello se exponen antecedentes de lo que constituyó la lucha de los movimientos estudiantiles mexicanos y su vínculo con los de América Latina, lo que permite ver que aún antes de la revolución mexicana, la efervescencia crítica y democrática de los estudiantes estaba ya presente.

En cuanto al periodo de la fundación (1925), las acciones y propuestas que Zuno planteó como proyecto fundacional, las características orgánicas de su modelo, se buscará mostrar cómo este no representó ninguna característica de soberanía respecto a Estado -limitando con ella su autonomía-, además de que se construyó con base a la marginación de los sectores universitarios que habían venido participando en el ámbito de la educación superior -alumnos y profesores-.

El trabajo se sustenta en elementos basados en el Análisis Institucional, desarrollado desde una perspectiva histórica planteando un acercamiento a los procesos instituidos del modelo confrontándolos con los efectos instituyentes, a fin de mostrar que la retórica y los criterios normativos sostenidos como la interpretación identificatorio del fundador Zuno y su grupo respecto al tipo de universidad, se revela realmente como una expresión caudillista, unilateral, verticalista en gran medida al analizar la conformación y la actuación final que desembocó en el modelo universitario. Con ello, constatamos que la fuerza de esa mística ideológica que envolvía con fuerza la atmósfera posrevolucionaria, limitó en gran medida construir un modelo universitario autónomo y democrático.

La metodología para este trabajo se basa en la consulta de fuentes secundarias y documentales, buscando que las evidencias que arrojen el análisis permitan ir más allá de la versión apologética y de efemérides que caracteriza la historiografía de la Universidad de Guadalajara.

\section{Antecedentes}

Producto de la importancia que adquirió la ciudad y su región, la Universidad de Guadalajara había sido fundada a finales de la época colonial en 1792. Fue la segunda Universidad en la Nueva España; de las últimas que se fundaron en las colonias españolas y de las pocas fundadas por Carlos IV. ${ }^{4}$ Este último aspecto es significativo, pues debido al estallido de la revolución francesa y las repercusiones que por ella se desataron en los siguientes años, influyó para que las reformas borbónicas ilustradas en España y en América hispana se inhibieran. La Universidad de Guadalajara lo resintió, de ahí que su modelo fundacional no contenía aquellas características que en el campo de la ciencia las universidades europeas e incluso españolas habían conquistado. ${ }^{5}$ Una vez consumada la independencia de México, la universidad fue suprimida en 1826 por el primer

4 Carmen Castañeda, La Educación en Guadalajara durante la Colonia. 1552-1821 (México: CIESAS, 2012). José Luis Zaragoza Raso, Crónica de la Real y Literaria Universidad de Guadalajara (Guadalajara: Instituto Jalisciense de Antropología e Historia, 1963). Juan B. Iguiniz, La Antigua Universidad de Guadalajara (México: UNAM, 1959). José Franco Cornejo, Documentos referentes a la fundación, extinción y restablecimiento de la Universidad de Guadalajara (Guadalajara: Universidad de Guadalajara, 1972).

5 José Luis Pezet, La Universidad Española. Siglos XVIII y XIX. (México: Editorial Taurus, 1974). 
gobierno liberal del nuevo Estado Libre y Soberano de Jalisco al considerársele retrógrada y representativa de la influencia religiosa y colonial.

En su lugar fue creado el Instituto de Ciencias, institución superior de tipo napoleónico que fungió también como universidad y que contenía ya carreras modernas, como Ingeniería. No obstante su carácter moderno identificado con la educación napoleónica, el Instituto no contempló una estructura participativa, como sí había sido incluso la Universidad colonial. ${ }^{6}$ Durante el siglo XIX sin embargo la Universidad fue reabierta de manera intermitente cuando los grupos conservadores tomaron el poder en Jalisco y cerrada cuando los liberales retomaban el gobierno y reabrían el Instituto. En 1860-63 ambas instituciones no volvieron a establecerse, quedando las escuelas superiores: Medicina, Leyes, Farmacia, Escuela Normal, Comercio e Ingeniería, operadas directamente por el gobierno del Estado de Jalisco a través de una Dirección de Estudios Superiores.

\section{La Revolución Mexicana: Impronta que marcó la Educación}

Después de un largo periodo dictatorial, la revolución irrumpió en 1910 como el primer movimiento social del siglo en el mundo. En 1917, después de un periodo convulso los revolucionarios establecieron, en una asamblea constituyente una constitución que contenía el reconocimiento de los derechos individuales, sociales y laborales de la sociedad, lo que implicó abrigar amplias expectativas de justicia social a través de reformas y transformaciones. Era un modelo de liberalismo social. ${ }^{7}$ Este acontecimiento impacto también la educación. En 1920 se inició el programa de la Escuela Rural Mexicana (ERM), modelo comunitario que implicó el establecimiento en todo lo ancho del territorio nacional de escuelas para educar, capacitar y apoyar social, cultural y educativamente a la infancia y a las comunidades particularmente rurales. ${ }^{8}$

Sin embargo, estas reformas revolucionarias no consideraron a la educación superior. La estrategia se concentró en la educación infantil y en la formación de maestros para la educación elemental. La Universidad Nacional de México, que había sido refundada al final del periodo dictatorial en 1910, gracias a la influencia de un grupo de positivistas encabezados por don Justo Sierra, literato y ensayista eminente, no resintió el impacto de la revolución y sus cambios; perviviendo en ella un modelo de Universidad basado en un humanismo universalista en

$6 \quad$ La Estructura orgánica del Instituto establecía que los nombramientos de director de la institución y de los profesores sería unilateral a partir de la voluntad del Gobierno del Estado. Artículos de la Ley de Instrucción para el Instituto del Estado de Jalisco. Colección de Leyes del Gobierno del Estado de Jalisco. En cambio en la legislación de la Real Universidad de Guadalajara.

7 Guadalupe Zuno, fundador de la Universidad, era un decidido convencido de la filosofía popular de la Revolución, su pensamiento e ideología política se puede apreciar en muchos de sus discursos y textos.

8 Existe una amplia bibliografía sobre la ERM, pues que es considerada la etapa más gloriosa de la educación en México, por su original modelo comunitario y por sus resultados. Algunos artículos que exponen algunos enfoques originales son: Lourdes C. Pacheco, "Fuimos a sembrar cultura. Los maestros y la construcción de la escuela rural mexicana", Investigación y Postgrado 28, No. 1 (2013). María Guadalupe Díaz Tepepa, "Técnica y Tradición. Etnografía-de la Escuela Rural y de su Contexto Familiar y Comunitario Investigación Administrativa", Investigación y Postgrado, No. 83 (1999). Justa Espeleta y Eduardo Weiss. "Las escuelas rurales en zonas de pobreza y sus maestros: tramas preexistentes y políticas innovadoras", Revista Mexicana de Investigación Educativa, No. 1, 1996. 
el campo de las ideas y del positivismo en el campo de las profesiones; ${ }^{9}$ configurándose un régimen de gobierno donde prevalecía su carácter autocrático y elitista. ${ }^{10}$ Esto influiría para que años después, en 1929, los estudiantes lucharan y conquistaran su autonomía y sentaran las bases de la libertad de cátedra. ${ }^{11}$ Estos elementos emblemáticos de su nuevo perfil, estaban sin duda influidos, además de sus propias problemáticas, por la gesta de Córdoba, en primer lugar porque cada cambio que se gestó en ella estuvo presidida por movimientos estudiantiles. ${ }^{12}$ En la Universidad de Guadalajara el camino fue diferente.

\section{Una Región, Una Ciudad, Una Gran Deuda Social}

Jalisco, provincia del occidente mexicano donde se asienta la ciudad de Guadalajara era un territorio de gran tradición productiva, tanto agropecuaria como comercial desde el periodo colonial. Fue además un centro político y religioso de gran importancia. Fueron establecidos en el siglo XVIII una imprenta, un obispado, un consulado comercial, una casa de moneda y una universidad, signos de su preponderancia. En el siglo XIX siguió caracterizándose por su gran empuje económico y político, siendo sin duda, su ciudad capital la más importante en términos demográficos, productivos, de servicios y como espacio político importante no solo en el occidente del país, solo superado por la ciudad de México.

Sin embargo, como sucede en todo el espacio latinoamericano, Guadalajara y su región también resintieron los resabios del modelo colonialista y las secuelas de una nación periférica con un desarrollo económico tardío y dependiente. De ahí que tanto en los siglos de dominación española como en el XIX y primeras décadas del XX, las condiciones de inequidad, pobreza, marginación, enfermedades y todas otras secuelas que trae consigo estos tipos de sociedades, propiciaron la pervivencia y crecimiento de clases subalternas con todo el corolario que arrastran histórica y socialmente. Se entiende entonces que al despuntar el nuevo siglo, se haya escenificado una revolución que entre otras cosas, además de pugnar por destruir la dictadura que imperaba por 30 años, abanderara causas

9 La Universidad de México según el modelo de su refundación en 1910, tenía autonomía relativa, pues como bien señaló Justo Sierra, su fundador. "nuestra universidad no es rigurosamente de Estrado...tiene personalidad propia...pero es el Estado quien la constituye...El Rector y el Consejo Universitario podían organizar los estudios, conferir grados académicos y adquirir bienes (administrar los recursos), pero el rector quedaba supeditado por el ministro de Instrucción pública y Bellas Artes que sería el jefe nato de la institución y sería nombrado por el presidente de la República..." Patricia Ducoing, La Pedagogía en la Universidad de México 18511954. Tomo 1. (México: CESU. UNAM, 1990), 94-95.

10 Habría según esta nueva normatividad fundacional, dos representantes de profesores y uno de los alumnos pero solo con derecho a voz. Esta situación propició la actuación regularmente arbitraria de funcionarios y profesores seguramente y detonó el movimiento autonómico y de representatividad de 1929. María de Lourdes Velázquez Albo, "El imaginario de la Universidad Nacional de México en 1910" (Ponencia presentada en el "X congreso Nacional de Investigación Educativa. Área 9, Historia e Historiografía de la Educación”, Veracruz, 21 al 25 de septiembre, 2009).

11 Renate Marsiske, "El Movimiento Estudiantil de 1929 y la Autonomía de la Universidad Nacional de México", Revista de Educación Superior 11, No. 44, (1982).

Diana Soto Arango y María Isabel, Lafuente. Autonomía y modelos universitarios en América Latina. Tomo II Bogotá, León. Universidad de León, España, Asociación Colombiana de Universidades. ASCUN. Universidad Pedagógica y Tecnológica de Colombia, RUDECOLOMBIA, 2007.

12 Ver al respecto la abundante bibliografía que sobre el tema de las reformas en la Universidad Nacional ha producido Renate Marsiske. 
para erradicar la desigualdad, la injusticia, la ignorancia y el atraso social. Estas banderas fueron las que sirvieron como recurso emblemático para la fundación de la nueva universidad. La revolución surgió en el norte del país y luego se intensificó en el sur. Jalisco no fue ajeno a esta lucha no obstante que en la región dominaba mayoritariamente el sector conservador quien apoyado por hacendados ricos y una iglesia militante.

\section{Nacionalismo, Revolución: Las banderas de la UDG}

En 1920, después de una cruenta etapa de violencia e instabilidad, se inició una etapa de reconstrucción nacional. En Jalisco, en 1923 sube a la gubernatura por el Partido Liberal Jalisciense don José Guadalupe Zuno Hernández, importante personaje que refunda la Universidad de Guadalajara en 1925. Liberal radical, con ideas nacionalistas, ferviente defensor del proyecto político de la revolución; artista e intelectual, es la cabeza de una pléyade de pensadores -políticos, escritores, profesores, artistas-, que agrupados en el "Centro Bohemio" que él funda, representan el pensamiento más avanzado de la revolución mexicana en Jalisco. ${ }^{13}$ Está ahora en el poder, un poder que necesita aprovechar ante las asechanzas de las otras facciones políticas que quieren derribarlo. ${ }^{14}$ Entre sus múltiples decisiones, decreta la fundación de la Universidad en 1925.

José Guadalupe Zuno fue un caudillo cultural y político. Basta ver por la forma como se gestó desde su acción unipersonal la Universidad de Guadalajara. Su grupo político e intelectual dio luz a una filosofía y una acción renovadora en el campo de la educación y la cultura. Cabría preguntarse, ¿qué es lo que encuentra Zuno como inspiración en la revolución mexicana para fundar una universidad identificada con ese movimiento? En realidad la revolución mexicana no estaba sustentada como la francesa o como la rusa en una ideología o doctrina política definida que la identificara diáfanamente. Es la conjunción de una serie de presupuestos políticos, banderas, ideologías, aspiraciones y acciones que dieron forma a lo que más tarde se llamó el nacionalismo revolucionario. ${ }^{15}$ También ha sido caracterizado como una ideología burguesa constituida desde un régimen de auto conciencia popular y reformista. ${ }^{16}$ Zuno ve la revolución como la más importante y estratégica oportunidad para redimir y educar al pueblo dentro de un sistema liberal democrático pero tutelado por un Estado fuerte.

13 Es interesante que a diferencia de otros grupos culturales importantes que existieron en ese siglo como la Generación del ateneo o el grupo de los contemporáneos, el Centro Bohemio conjunto artistas, intelectuales y políticos en activo. José Guadalupe Zuno Hernández, Anecdotario del centro bohemio (Guadalajara: Pedro Rodríguez Lomelí, 1964). Participaron muy activamente en las juntas preparatorias a la restauración de la Universidad, además del ingeniero Agraz, del profesor Díaz de León y del gobernador Zuno (quien las convocó y las presidió), las profesoras Irene Robledo García y Catalina Vizcaíno, el arquitecto Agustín Basave del Castillo Negrete, los presbíteros Severo Díaz Galindo y José María Arreola Mendoza, el doctor Juan Campos Kunhardt, el abogado Ignacio Villalobos Jiménez y los profesores Adrián Puga Gómez y José Ignacio Calderón Bonilla.

14 Especialmente desde la presidencia de la República, donde el presidente, que pertenecía a otra facción política buscaron destituirlo. Ver: Jaime Tamayo Rodríguez, "El enfrentamiento Zuno-Calles", en la Revolución en las Regiones, Vol. 2 (Guadalajara: Universidad de Guadalajara, 1986).

15 Ver: Ramón Eduardo Ruiz, La gran rebelión. 1905-1904, (México: Editorial Era, 1982).

16 Arnaldo Cordoba, "México. Revolución burguesa y política de masas", Cuadernos políticos, No. 13 (1977). 
No se trata de una transformación radical del modelo social sino de una gran reforma, estableciendo instituciones que le permitan a la sociedad derechos en el trabajo y a su capacitación técnica pues atribuye el atraso del país a la falta de oportunidades, a su sobrexplotación laboral, producto esta última de la ignorancia y falta de preparación. ${ }^{17}$ Otro elemento identificatorio de su pensamiento y del de muchos de los líderes revolucionarios de ese tiempo es su anticlericalismo. ${ }^{18}$

El Estado, que ha emergido de la revolución debe reconstruir el modelo social y productivo y desde luego el educativo, reconociendo en la sociedad una capacidad febril, creatividad, imaginación y talento. Lo que falta son instituciones y leyes que impulsen el trabajo y la escuela. El discurso de un ingeniero recién llegado de Europa, Juan Salvador Agraz, ${ }^{19}$ le permite vislumbrar a Zuno la necesidad de apostar por la educación, haciéndolo desde la educación superior que tenga como fundamento la diversidad de profesiones necesarias para el desarrollo productivo. ${ }^{20}$ Él le agregaría una orientación popular y esté cobijada bajo la coraza de una universidad vinculada a la sociedad, pues de esa manera el Estado proporcionará una educación laica, científica, capacitadora, sustentada en un Estado revolucionario orientado al progreso social.

\section{El Contexto Fundador: Una Universidad de Estado}

A diferencia de la mismísima Universidad de Córdoba que gestó sus cambios desde abajo, es decir desde el estudiantado cuando en 1919 su rebeldía enfrentó una élite jesuítica y oligárquica que dominaba monolíticamente la institución tanto con un discurso dogmático como con un currículo desfasado, así como por la intervención autoritaria del Estado; o como la Universidad de México donde también los estudiantes enfrentaron una actitud dictatorial y monolítica de profesores y autoridades en 1929, la Universidad de Guadalajara en cambio, gestó su modelo desde arriba, es decir, impulsado por el Estado, o de un sector poderoso del Estado, aquel que, estando en el poder en Jalisco, estaba representado por el gobierno zunista.

17 Zuno es elocuente en definir constantemente sus motivaciones, interpretaciones y acciones en las que participó precisamente porque fue un escritor prolífico que registró y publicó su vida política. Ver: sus discursos y su obra autobiográfica. En este texto se incluyen algunas referencias.

18 En gran parte de sus discursos y escritos antes de que fuese gobernador expresa su radicalidad anti iglesia. Ya como gobernador atempera sus expresiones por razones políticas y de diplomacia y porque busca tener aliados en la localidad ante las asechanzas que sobre él ejerce el gobierno central del presidente Plutarco Elías Calles. Una vez que dejó el poder vuelve a mostrar su pensamiento anticlerical. Ver su obra organizada en varios tomos: José Guadalupe Zuno, Reminiscencias de una vida (México: El Diario, 1972).

19 El Ing. Agraz, producto de sus estudios en Francia y Alemania, tenía una amplia visión científica enfocada a convertir a México en un país líder de la producción de patentes a través de centros de investigación científica vinculados o creadores de un aparato productivo fuerte y soberano en el campo de la Química. Sus ideas no fueron comprendidas y abandonó finalmente las reuniones para la fundación de la Universidad. Había sido antes el primer director de la Escuela de Ciencias Químicas de la Universidad Nacional. (1916-1918).

20 En lugar de promover el desarrollo de la investigación, la universidad incluyó como parte de su estructura la educación universitaria politécnica, primera en toda América Latina. “Expediente: Leyes y reglamento ¿¿de qué? 1994-2010”, (Ciudad), (LOUG. AHUDG) Escuela Politécnica. 
Este hecho es además significativo puesto que esta reforma fundacional, progresista, laica y oficial, gravita en una sociedad donde pervive un amplio sector poderoso representado por un empresariado urbano y rural activo pero vinculado férreamente a la Iglesia, y en este sentido una sociedad muy apegada a las ideas confesionales y hasta fanáticas, evidenciado en un apego cotidiano y enfático a los dictados de una iglesia local belicosa y doctrinal. Es decir un ambiente social retrógrado, sin dejar de reconocer la existencia de grupos de avanzada entre maestros, obreros, estudiantes y clubes políticos ${ }^{21} \operatorname{como}$ el Centro Bohemio referido. Esta situación refleja la valentía, arrojo, visión y originalidad del proyecto de Zuno y su gobierno. ${ }^{22}$

Guadalajara era una ciudad que producto de la guerra no había perdido gran parte de su capacidad económica. Pero se manifestaba una profunda desarticulación en sus fuerzas productivas; en sus canales comerciales y un gran desfase en servicios. Sí, era ya una ciudad grande, pero desigual, y con una burguesía anodina profundamente inequitativa y en donde "las continuidades entre el antiguo régimen y el nuevo, producto del movimiento revolucionario, sobresalen por encima de las rupturas introducidas en el seno de la sociedad por este último, rupturas que solo tendrán lugar de manera lenta y pausada, al cabo de un dilatado período de incubación, y sin eliminar del todo las continuidades. ${ }^{23}$ Jalisco a la hora de la fundación de la Universidad de Guadalajara está gravitando donde los grupos económicamente más poderosos están además cohesionada por las fuerzas de la religión. En esta composición gravitaban sectores liberales radicales y revolucionarios que habían llegado finalmente al poder, primero con la llegada de Manuel M. Diéguez, quien podía considerarse el sector más genuino de la revolución ${ }^{24}$ y luego con la coyuntura electoral en 1924 al llegar Zuno al gobierno del Estado. ${ }^{25}$

\section{Visibilidad e Invisiblidad de los Estudiantes}

Desde antes de los acontecimientos de Córdoba, que pronto se difundieron por toda latinoamerica, las Universidades y los estudiantes se organizaban, se asociaban y discutían los asuntos relativos a las distintas problemáticas uni-

21 Un ejemplo emblemático de la existencia de personales o grupos revolucionarios en Jalisco es del de Roque Estrada, quien estudió la preparatoria y la carrera de abogado en Guadalajara. Se tituló en 1906. A pesar de su juventud se dio la tarea de organizar a los obreros. Fue partidario del magonismo desde 1909, que fue la corriente más importante contra la dictadura antes del estallido revolucionario, fue expulsado por Miguel Ahumada gobernador del Estado. AHUDG. Carpeta. Expedientes de Profesores. Ver también a José Ramírez Flores, La revolución maderista en Jalisco (Guadalajara: Universidad de Guadalajara, 1992).

22 José Guadalupe Zuno Hernández, La historia de la revolución en el Estado de Jalisco (México: Instituto Nacional de Estudios Históricos de la Revolución Mexicana, 1964). Las reformas del Callismo y de Diéguez, y Zuno en Jalisco, desató una reacción histérica y violenta como fue la guerra cristera cuyo foco principal fue Jalisco entre 1926 y 1929. Soledad Loaeza, "La rebelión de la Iglesia", Revista Nexos (Junio de 1984):11-17.

23 Rafael Torres Sánchez, Jalisco y la Revolución mexicana. Estructura, Economía y Cambio Institucional (México: Universidad Autónoma de México, 2001).

24 La gubernatura en Jalisco producto del movimiento constitucionalista (1914-1919), significó la llegada al poder de un gobernante del militarismo revolucionario, pero de pensamiento radical en el campo de su política social, obrera e incluso educativa. Mario Aldana Rendón, Manuel M. Diéguez y la revolución mexicana (Jalisco: El Colegio de Jalisco, 2006).

25 Jaime Tamayo, “Zunismo y movimientos sociales” y "El enfrentamiento Zuno-Calles” en La revolución en las regiones. Vol. 2 (Guadalajara: Universidad de Guadalajara, 1986). 
versitarias y escolares, e incluso políticas. ${ }^{26}$ Muchas de ellas eran semejantes, lo que ayudó a la cohesión de un movimiento continental para aquellos años. En septiembre de 1921 se reúne en México el Primer Congreso Internacional de Estudiantes, ${ }^{27}$ surge de allí la Federación Internacional de Estudiantes. Sería ilógico pensar que este importante evento celebrado en México no haya tenido eco en Guadalajara. El discurso inaugural lo da el rector de la Universidad de México: José Vasconcelos.

"La delegación argentina estaba formada por estudiantes de gran prestigio en su país, Héctor Ripa Alberdi, presidente de su delegación, era miembro de la Junta Representativa de la Federación Universitaria Argentina, presidente del Colegio Novecentista, redactor de "Renovación" órgano de la Federación Universitaria de la Plata, había publicado un libro de poemas: "Soledad" y era prestigiado escritor. Arnaldo Orfila Reinal, había sido presidente del Comité de la Huelga Grande de la Plata, Secretario del Comité Pro-afianzamiento de la Reforma Educacional y de la Asociación de Ex-alumnos de la Universidad de la Plata, tenía una larga actuación en el periodismo estudiantil y era profesor de la Universidad Obrera. Vrillaud había sido presidente de la Federación Universitaria de Santa Fe y del Centro de Estudiantes de Derecho y uno de los primeros líderes de la campaña social que se realizó en su tierra entre 1919 y 1920. Y Dreyzin como los anteriores tenía gran ejecutoria estudiantil y periodística." 28

Vemos que la rebeldía estudiantil está latente en toda América Latina, en donde los estudiantes estaban en una franca etapa de organización y discusión continental sobre las universidades y las sociedades de América.

Los estudiantes tapatíos ${ }^{29}$ desde más de dos décadas, no obstante que no había universidad sino solamente instituciones de educación superior dependientes del Gobierno del Estado, tuvieron siempre una estirpe rebelde, irreverente y contestataria, llegado a constituir organizaciones bien conformadas y representativas. $^{30}$

26 En plena dictadura porfirista las acciones estudiantiles fueron frecuentes en México. En 1910, se organizó el Primer Congreso Nacional de Estudiantes en el cual participaron delgados estudiantiles de Guadalajara Ver: Javier García Diego, "Movimientos estudiantiles durante la revolución mexicana (Estudio de caso de la participación de un grupo de clase media urbana)" en Los estudiantes, trabajos de historia y sociología, eds. Renate Marsiske (México: CESU-UNAM, 1989), 144.

27 El evento fue convocado por la Liga Panamericana de Estudiantes con representantes de muchos países, entre ellos Uruguay y Argentina del cono sur. En México lo organizó la Federación de estudiantes del DF, que encabezaba Daniel Cosío Villegas -el cual llegaría a ser años después un gran historiador político-. Por la Federación de Estudiantes Argentinos asistieron: Héctor Ripa Alverdi, Arnaldo Orfila Reinal, Pablo Vrillaud y Enrique Dreyzin. Por México, Daniel Cosío Villegas, Raul J. Pous Ortíz, Rudolfo Brito Foucher, Francisco del Río y Cañedo y Miguel Palacios Macedo. Participaron también por México como delegados adherentes, entre otros: Ramón Beteta Quintana, Manuel Gómez Morín, Vicente Lombardo Toledano Luis Enrique Erro y Alfonso Caso, que llegarían a distinguirse como importantes intelectuales e investigadores años después. También participaron por Centroamérica, Rafael Heliodoro Valle, Miguel Ángel Asturias y Salomón De la Selva y Pedro Enríquez Ureña, de Santo Domingo, quienes también después fueron importantes escritores y humanistas.

28 Ciriaco Pachecho Calvo, "El Primer congreso internacional de estudiantes celebrado en México en 1921", Revista Universidad de México, Tomo III, No. 14 (Diciembre de 1931): 187.

29 Oriundos o vecinos de la ciudad de Guadalajara, Jalisco, México.

30 Desde que las Facultades pasaron a ser parte del organigrama del gobierno del Estado al cerrarse el Instituto y la Universidad, los estudiantes sufrieron de la actitud vertical, unipersonal y arbitraria de profesores y directivos. Es frecuente encontrar peticiones de cambios, reclamos y solicitudes de remoción de profesores. Hay incluso movilizaciones. Las agrupaciones fueron por escuela hasta 
La declinación del General Reyes a la presidencia de la república en 1919 y por la cual se había conformado un comité estudiantil de apoyo y anti dictatorial, no significó su disolución, por el contrario, los estudiantes se politizaron al darse cuenta que Reyes era incondicional del dictador Díaz. Ante la proliferación de manifestaciones la policía intervino y hubo represión a todos los reeleccionistas y a la población estudiantil. Estos, formaron un comité "La liga estudiantil de Guadalajara" y trasladaron a la ciudad de México para solicitarle personalmente a Díaz que detuviera la represión y liberara los inconformes. ${ }^{31}$

Escudriñando en los años previos a la fundación de la universidad de Guadalajara (1919-1925), no he encontrado documentos universitarios, ni gubernamentales, ni tampoco personales (de don José Guadalupe Zuno o del grupo fundacional que lo acompañó), que hagan mención expresa sobre los hechos de Córdoba. ${ }^{32}$ Tampoco sobre la autonomía como concepto institucional para impulsarla desde una perspectiva de autogobierno o de soberanía académica y de territorialidad. En realidad, Córdoba no está presente en el imaginario fundacional como referencia expresa. Sí lo está, en algunos de los aspectos que le dieron a Córdoba su legitimidad constitutiva como institución de Estado cuya majestad era la presencia de la educación pública superior articulada en una Universidad y bajo un modelo técnico-popular, pero no precisamente reivindicando la presencia de una cultura democrática de participación docente ni mucho menos estudiantil no obstante que Guadalajara tenía una larga historia de movilizaciones estudiantiles. ${ }^{33}$.

Cuando menos desde 1910 se gestaba ya la participación de estudiantes de Guadalajara respecto a nuevas ideas políticas y artísticas. ${ }^{34}$ En efecto, años antes, no obstante la dictadura porfirista, en Guadalajara, los estudiantes del Liceo del Estado realizaron acciones anti dictatoriales. El 14 de abril de 1908 organizaron una Sociedad de Estudiantes y eligieron como su primer presidente a Javier Enciso. Durante la campaña presidencial de 1909, estos estudiantes apoyaron la candidatura de Bernardo Reyes, formando una de las primeras organizaciones estudiantiles de carácter político: la Liga de Estudiantes de Guadalajara, cuyo primer presidente fue Guadalupe González. ${ }^{35}$ En 1910, los estudiantes de Guadala-

que en 1909 se establecieron organizaciones representativas de todas y cada una de las escuelas. Ver: "Incidencias escolares y de materias. 1901-1909" (Guadalajara), Archivo Histórico de la Universidad de Guadalajara (AHUG), Sección de minuta y correspondencia. 1900 a 1923.

31 Guadalajara, AHPD, I 34.c 20. Doc. 9626-27 y 13549. Los estudiantes detenidos fueron expulsados por "exceso de faltas a clases".

32 José Guadalupe Zuno, Reminiscencias, op. cit., (cita 17). En el AHUG, en las carpetas de correspondencia ni en las actas, alguna mención sobre los acontecimientos de Córdoba.

33 Alfredo Mendoza Cornejo, Organizaciones y Movimientos Estudiantiles en Jalisco de 1900 a 1937. (Guadalajara: Universidad de Guadalajara, 1989).

34 La gestación de las nuevas ideas estaban ya latentes por quienes siendo entonces estudiantes impulsaban ya sus nuevos proyectos. Según el extinto profesor don Nacho Martínez: "En Guadalajara, (en 1912) un grupo de estudiantes revolucionarios tomaba destacada participación en la política y al tiempo que intervenía en la elaboración de las ideas sociales (...) entre ellos: Xavier Guerrero, Joaquín Vidrio, Alfredo Romo, José Guadalupe Zuno, Enrique Díaz de León, Amado de la Cueva y Juan Antonio Córdova." Ignacio Martínez, Pintura Mural Siglo XX. Jalisco en el Arte (Guadalajara: Editorial de Planeación y Promoción, 1960). Vemos aquí que de nuevo son artistas y políticos quienes unidos participan con visiones más integrales de sus propuestas.

35 Ver: Alfredo Mendoza Cornejo, op, cit., (cita 33).También: Schulamit Goldsmit Brindis, "Jalisco", en Contento y descontento en Jalisco, Michoacán y Morelos 1908-1911, eds. Graciela de Garay y otros (México, UIA, 1991), 27. 
jara habían realizado movilizaciones contra Estados Unidos, lo que mostraba ya su incipiente organización. ${ }^{36}$

En ese mismo crucial año, en el Primer Congreso Nacional de Estudiantes de la República, celebrado en la ciudad de México, participaron por Jalisco: Enrique S. Pérez Arce -de la escuela de Jurisprudencia-, y Salvador Jiménez Loza -Escuela de Ingenieros Libres- ${ }^{37}$ Está también la participación del brillante líder estudiantil tapatío Salvador Navarro Aceves, cuyas "actividades revolucionarias... se desarrollaron, sobre todo en la Universidad de Guadalajara, donde perteneció a lo más selecto de la vanguardia. Fue representante de su Universidad en tres congreso de estudiantes y en todos ellos tuvo una magnífica labor" ${ }^{\prime \prime 8}$. Don José Ramírez Flores, logró obtener las actas de las decenas de reuniones clandestinas que diferentes ciudadanos, obreros, empleados, estudiantes organizaban en Guadalajara previo al levantamiento revolucionario. Las reuniones de estudiantes muestran un nivel de organización y participación constante. En 1910, se consigna:

Por acuerdo del C. Enrique Ramírez Calleros, el presente libro usado y que empieza en el folio No. 24 se dedica a la secretaria del club anti reeleccionista "Valentín Gómez Farías", por lo tanto entréguese el C. Eduardo J. de la Torre para que en él haga las anotaciones de las sesiones y sesiones, entretanto se recoge al C. Manuel Alvarado Mendoza el libro de las actas y los documentos que guarda en su poder. Sufragio Efectivo No Reelección. Guadalajara Jalisco 15 de Enero de 1910. El segundo Secretario de la Sociedad de Estudiantes de Guadalajara. ${ }^{39}$

Así que a diferencia de la opinión muy difundida de que Jalisco y en especial Guadalajara había sido un enclave retrógrado que no solo no participó en el movimiento de 1910 sino que se opuso a él, en realidad la ciudad tenía una gran agitación política y había constantes grupos conspiratorios, protestas y reclamos antes y después del movimiento armado. Los estudiantes dan cuenta de ello. Como queda consignado entre otros episodios con el periódico "Juventud Liberal" órgano del partido Liberal que actuaba activamente en Guadalajara. Este órgano consigna la formación del "Partido Liberal Estudiantil" que surgió para oponerse al Partido Católico que había ganado las elecciones en $1912 .{ }^{40}$ Este partido estaba conformado por estudiantes de las carreras profesionales y del Liceo de Varones de Jalisco. ${ }^{41}$

Así que la influencia de la participación estudiantil estaba presente. Agreguemos ese trascendental hecho universitario: Córdoba, que fue la epopeya que detonó la irrupción de la universidad moderna desde su perspectiva democrática,

36 Servando Ortoll, “Turbas anti yanquis en Guadalajara en vísperas de la revolución del diez" Boletín histórico de Jalisco 1, 2a. epoca, No. 2 (Mayo agosto 1983): 2-15.

37 Rafael Heliodoro Valle y otros, Añoranzas del primer congreso de Estudiantes (1910), (México: Instituto de Historia de Nicaragua y Centroamérica, 1943), 118.

38 Humberto Salvador, prólogo a El movimiento artístico de México, por Salvador Navarro Aceves, (Quito: Editorial ELAN, 1935$), 15$.

39 Ramírez, Op cit. Pág. 129.

40 Ver: el periódico "El Correo de Jalisco" correspondiente a los años 1912 al 1914.

41 Alfredo Mendoza Cornejo, op. cit., (cita 33), 96. 
soberana y autogestiva, reivindicando la conquista de un modelo emancipatorio en su régimen y en su filosofía académica a gran parte de las universidades de América e incluso Europa. Pero estos elementos, no tuvieron eco en Guadalajara, o no se reflejan diáfanamente, seguramente porque no se difundieron los acontecimientos ni el manifiesto de Córdoba. ${ }^{42}$ La ausente preocupación por la autonomía ni por otros elementos constitutivos de lo que fue la lucha cordobesa fue considerada entonces sino hasta muchísimos años después, ${ }^{43}$ cuando sin cambiar su nombre la universidad de Guadalajara buscó entonces sí su autonomía plena debido a su dependencia orgánica al gobierno del Estado tal como don Guadalupe la concibió y fundó.

\section{Fundación de la Universidad}

Cuando ya tenía el propósito de fundar la universidad, el gobernador Zuno tuvo la fortuna de realizar una serie de reuniones con diferentes amigos y profesionistas que contribuyeran a delinear el tipo de universidad que se iba a fundar. Pero además tuvo también el detalle de que se redactasen las actas de todas y cada una de las reuniones, lo que posibilita que podamos ver como se fue configurando el modelo de la universidad. Las actas las publicó en su autobiografía ${ }^{44}$ Estos documentos son claves para conocer las motivaciones y argumentos que se dieron para delinear el modelo de Universidad a fundar. Julio Acero rememora los motivos que dieron lugar a la fundación de la Universidad por parte del grupo que convocó Zuno:

Se analizaron todos los aspectos desde la organización histórica de los planteles de enseñanza superior en México y en Nueva Galicia; se conformó y debatió un esquela muy avanzado de la Universidad que daba ala Tecnología y a los problemas socio-económicos una ubicación universitaria y un contenido que con anterioridad apenas se esbozaba, se clasificaron las Facultades, las escuelas adherentes a ellas y las instituciones nuevas; se estudiaron los temas de la extensión de los estudios, las condiciones y prerrogativas de la Docencia, así como las que corresponderían al alumnado. ${ }^{45}$

Tal vez la anterior motivación fundacional pueda también entenderse por lo que se omite. Es decir: la forma como sería gobernada la universidad, lo cual quedó explícito cuando Zuno presentó redactada la Ley orgánica en donde su autoridad moral le permitía su decisión unipersonal de decidir sobre este asunto. En una reflexión hecha años después, Don Guadalupe al abordar el problema de la representatividad en el gobierno de la institución dice:

42 En una revisión parcial de la prensa en Guadalajara de la época no encontré notas periodísticas ni editoriales sobre el movimiento de Córdoba en la etapa de su insurgencia y desenlace.

43 El gobernador Jesús González Gallo (1947-1953) a final de los años cuarenta, intentó convertir a través de una nueva ley orgánica a la universidad en un simple departamento del gobierno del Estado, aprovechando la dependencia jurídica de la Universidad al gobierno desde la época de Zuno. Situación que no prosperó pero que ocasionó un conflicto estudiantil e institucional.

44 José Guadalupe Zuno, Reminiscencias de una vida. Tomo I. (Guadalajara: Biblioteca de Autores Jaliscienses, 1956).

45 “Palabras de Ramón Córdoba”, Revista de la Universidad de Guadalajara, No. 13 (1966). 
La Universidad de Guadalajara, la actual, fundada en 1925, sobre cimientos limpiamente populares, congruente con ese espíritu revolucionario, formó su consejo General Universitario, con representaciones del profesorado y de las agrupaciones estudiantiles y con ello da un ejemplo a otras instituciones mexicanas, que han permanecido negadas a la juventud su entrada a las altas esferas académicas y de organización general que tanto le atañen. ${ }^{46}$

Las circunstancias parecieron mostrar otra cosa. En las reuniones con ese consejo de notables que delinearon el tipo de universidad así como para formar después el Consejo Universitario, Zuno no tomó en cuenta a la Confederación de Estudiantes de Jalisco, ${ }^{47}$ organización que agrupaba la representación del estudiantado en las escuelas superiores, la escuela Preparatoria y la Escuela Normal y que en 1923 se confederó para poder encarar el aislamiento y la dispersión del estudiantado. A la hora de designar a los representantes estudiantiles para el Consejo Universitario, se designaron alumnos conforme a la propuesta del gobernador. Con ello, se desconoció toda la tradición de lucha del estudiantado tapatío.

Para entender mejor los propósitos fundacionales, es importante añadir que si el inspirador principal de la Universidad fue Guadalupe Zuno, hay otro personaje que lo acompañó como orador y guía académico para la fundación de la Universidad: Enrique Díaz de León, quien muestra también las motivaciones que llevaron a cristalizar en el proyecto universitario. En las biografías que sobre él se han elaborado, se abona su importante participación antes y como primer rector en 1925 y responsable de conducir todo el proyecto institucional e ideológico de la institución. Todo parece indicar que la motivación que inspiró la creación y modelo tiene sus fundamentos en causas internas: nacionales y locales, propiciadas por la necesidad de impulsar para la sociedad, los nuevos proyectos emanados de la revolución mexicana, tanto en el campo de un nuevo régimen político como en una educación popular, profesional y técnica, cuya esfera debía constituir el fundamento político e ideológico nacionalista, laico y estatal de la educación superior. El análisis del pensamiento y acción de Díaz de León así lo muestra. $^{48}$

Un elemento importante que caracteriza la singularidad de la fundación de la Universidad es el hecho que el sustento de las críticas y banderas de Córdoba relativos a las condiciones internas en las que vivía esa Universidad, en Guadalajara no se apreciaban de manera aguda, puesto que no había universidad para esos años (1865-1924), sino escuelas profesionales dependientes del Gobierno del Estado de Jalisco, lo que significó que las problemáticas que había en cada una de ellas, se manifestaban de manera aislada, dispersa. Los estudiantes no

46 José Guadalupe Zuno, prólogo a Biografía de Prohombres Liberales, (Guadalajara:1970), 8.

47 Alfredo Mendoza Cornejo, Universidad de Guadalajara. Historia. Antecedentes, fundación y Devenir. 1696-1994. (Guadalajara: Ediciones del autor, 2005), 57

48 Mario Aldana Rendón, “Enrique Díaz de León y su circunstancia política” en Enrique Díaz León: Filosofía y Acción Política, eds. Mario Aldana Rendón y Miguel Romero Morett, (Guadalajara: Universidad de Guadalajara, 1988), 11-55. 
tenían una identidad institucional, lo que hacía que sus problemas y reclamos los realizaran de manera unilateral, ${ }^{49}$ hasta que en 1923 estaban ya agrupándose de manera representativa.

A su vez, en los motivos de la refundación de 1925, está muy marcada la necesidad de ajustar cuentas al pasado; Zuno busca rescatar el nombre de Universidad para la educación pública y sustentada en una concepción de progreso. Así lo rememora:

Le llamamos a esta casa Universidad porque ella es congruente con la intención de universalidad, abandonando el nombre de Instituto que restringe con el solo enunciado su campo de gestión en el acrecentamiento dela cultura. No nos detuvo el hecho histórico de que en nuestras luchas pasadas los liberales tomaran por bandera al Instituto y los conservadores a la Universidad. Aquello quedó liquidado en el campo político y no lo llevamos más allá, porque sabíamos muy bien que, entre lo más valioso del botín quitado al enemigo estaba precisamente la Universidad. En ella caben todos los Institutos, en ellas todas las ramas del conocimiento humano y de la investigación tienen su lugar natural e inclusive los intereses culturales de los vencidos. ${ }^{50}$

Las motivaciones históricas están unidas a su concepción ideológica de izquierda oficialista. ${ }^{51}$ En sus motivaciones la situación por restaurar para la educación superior pública una Universidad era para él fundamental, porque la potestad universitaria da un estatus institucional y de identidad reconocida por su majestad y linaje histórico. Se distingue en este concepto el que la universalidad esta uncida a una ideología y una filosofía centradas en un pensamiento que reivindica la condición de redención hacia los sectores más desfavorecidos, buscando que quienes alcancen a ocupar sus aulas se impregnen de una filosofía identificada con esa condición, con su cultura popular y con la necesidad de contribuir a la transformación justa del país. De ahí que cualquier paradigma que esté disfrazado de ideas aparentemente de pluralidad y que intente convertirse en fundamento de conocimiento del mundo y de la vida social, era absolutamente rechazado:

Porque allá (en otros establecimientos universitarios) se les tendrá siempre como parciales, ya que de suyo y voluntariamente son intolerantes y llaman libertad de cátedra a la imposición de un solo credo religioso, político y social. Nosotros somos más

49 Desde el siglo XIX no faltaron los reclamos, exigencias e inclusive paros y peticiones de estudiantes del Liceo y de educación superior respecto a lo que sucedía en sus escuelas, pero no pueden considerarse como auténticos movimientos estudiantiles, con un programa y una organización de conjunto, sin dejar de reconocer que mostraron siempre su actitud de rebeldía y conducta contestataria. Ver: Alejandro Mendoza Cornejo, op. cit., (cita 46).

50 José Guadalupe Zuno, “Discurso pronunciado en el XXXIII aniversario de la fundación de la Universidad de Guadalajara”, en Reminiscencias de una vida, eds. José Guadalupe Zuno (Guadalajara: Biblioteca de autores jaliscienses, 1958), 173-188.

51 La idea de socialismo e izquierda en los primeros gobiernos de la revolución mexicana coquetea continuamente como auto calificación de parte del propio régimen o como señalamiento analítico de parte de una historiografía oficialista. Las reformas sociales son muestra de un socialismo latente que avanza. Es un socialismo o un izquierdismo que se conceptualizó finalmente en el modelo educativo del cardenismo (1934-1940), cuyas fulgurantes luces ideológicas se expresaban ya desde las discusiones del constituyente de 1917. Ilán Semo, "El cardenismo revisado: la tercera vía y otras utopías inciertas". Revista Mexicana de Sociología 55, No. 2 (1993). Gilly Adolfo, "Los Dos Socialismos Mexicanos". Revista Nexos (1986). 
ambiciosos, porque aquí tenemos todo, todo respetamos y todos aquí hablamos y decimos nuestra verdad, alumnos y maestros. Los laboratorios funcionan sin limitaciones, las investigaciones se hacen si atender ningún dogma, sin técnica y científicamente, es decir somos depositarios y promotores de una verdadera cultura general, objeto real de nuestra Universidad. ${ }^{52}$

El modelo universitario sustentado en esta visión reivindicadora y caudillista, sustentado en un discurso efectivamente progresista y orientado a una doctrina de una determinada idea de izquierda de aquella época, tenía como virtud una aplicación irrestricta como modelo porque se ejecutaba desde la capacidad ejercida por el Estado; y fue sin lugar a dudas un gran avance si pensamos en las condiciones tan retrógradas que había en el Estado, pero era también un modelo restrictivo, limitado y contradictorio porque estaba construida desde una visión del poder, unilateral, de ahí que desde su inicial funcionamiento no fue nunca un dechado de democracia y participación porque la política universitaria se ejecutaba desde las cúspides de la dirección rectoral establecida conforme una jerarquía superpuesta (gobernador, rector). Y cuando hubo necesidad de que se integraran a las decisiones del poder universitario la voz y representación estudiantil de manera más orgánica y soberana, acorde con lo que sucedía en otras universidades, esta representación no fue concebida en la estructura deliberativa y de gobierno de la institución (Consejo Universitario). Esta decisión ha trascendido el tiempo y puede entenderse como una premonición, pues desde entonces las organizaciones estudiantiles han sido concebidas y controladas por el propio poder político universitario. ${ }^{53}$

La Confederación de Estudiantes de Jalisco, (CEJ) se había constituido en esos años en una organización vigorosa, activa y con propuestas innovadoras. ${ }^{54}$ Ha sido tal vez la única organización estudiantil independiente en Jalisco, la cual funcionó paradójicamente cuando aún la Universidad no existía. Paradójico también es que no haya luchado por formar parte del gobierno representativo de la Universidad cuando esta se gestaba y que una vez fundada se haya extinguido. ${ }^{55}$ Tal vez la fuerza política legitimadora y con un matiz revolucionario del fundador Zuno que no aceptó ninguna sombra sobre sus decisiones fundatorias, haya avasallado a la CEJ.

52 Discurso, op. cit., (cita 50).

53 Todas las organizaciones estudiantiles a partir de la fundación de la Universidad fueron concebidas, fundadas y controladas desde entonces por el propio poder universitario: FEJ, FESO, FEG, FEU, a excepción de las organizaciones que siendo independientes se lanzaron a la huelga de 1933 contra la Rectoría. Un breve estudio imparcial sobre esta huelga, ver: Enrique Francisco Camarena, "La primera huelga de estudiantes universitarios" en Narraciones Tapatías, De Guadalajara Jal. de 1900 a 1958 (Guadalajara: Librería el estudiante, s.f), 237-242. Están también los trabajos del prolífico Alfredo Mendoza Cornejo que escribió cuatro libros sobre "Organizaciones y movimientos estudiantiles", 1900-1937 (1989), 1935-1948, (1990), 1948-1954 (1992), 1954-1963 (1993), op. cit.,

54 Dice Mendoza C.: "como gran ausente de esa trascendente labor quedaron los estudiantes, que a pesar con una agrupación que para esos momentos los representaba legítimamente no fueran llamados a las reuniones de trabajo a fin de expresar sus puntos de vista." Mendoza, op. cit., 1900-1937.

55 Revisar las noticias sobre la CEJ que aparecían regularmente en los periódicos locales como "el Sol". Ahí se da cuenta de sus acciones, propuestas y opiniones, como la formación de una "Sociedad Científica Estudiantil” (El Sol: 13 de febrero de 1925), Presentación de dos proyectos: "Convenio con los doctores de la localidad para la atención médica barata a los estudiantes de educación superior, así como la fundación de una "botica estudiantil" a través de una cooperativa que ofrecía medicina barata a los estudiantes. (El Sol: 2 de marzo de 1925). 
Lo mismo sucedió con los profesores, que no tuvieron representación gremial ni mutualista sino hasta que las propias autoridades las fundaron muchos años después. ${ }^{56}$ Pero este fenómeno es asunto de otro ensayo, lo cierto es que a diferencia de Córdoba donde la participación estudiantil fue siempre soberana, independiente, autogestiva, en la Universidad de Guadalajara esta influencia no cristalizó; lo que inauguró un corporativismo caudillista, pues desde entonces las organizaciones estudiantiles quedaron sujetas a una correa de transmisión directiva, sin soberanía.

En cuanto a su autonomía, el origen autoral del gobierno en turno que fundó la institución marcó una dependencia de ella el propio gobierno del Estado, pues el rector era designado por el gobernador según la ley orgánica establecida y a su vez los directores de las escuelas superiores eran designados por el rector. Toda una cadena. Un evidente vínculo vertical que hizo estar a expensas durante muchos años a la universidad de los gobiernos en turno, lo que propició una contante lucha entre autoridades y las representaciones estudiantiles que para entonces habían ido formado conjuntamente grupos de poder corporativo y caciquil a fin de enfrentarse al Estado cuando fue necesario.

Finalmente el 7 de septiembre de 1925, el Congreso del Estado de Jalisco autorizó al gobernador Guadalupe Zuno para que expidiera la Ley Orgánica de la Universidad, la cual fue promulgándola el 25 del mismo mes. Las dependencias universitarias que empezaron a funcionar fueron: Facultad de Medicina, Facultad de Jurisprudencia, Facultad de Comercio, Escuela Preparatoria de Jalisco, Preparatoria para Señoritas, Facultad de Farmacia, Facultad de Ingeniería, Escuela Normal Mixta, Observatorio Meteorológico del Estado de Jalisco y la Biblioteca Pública del Estado.

Pocos días después la ceremonia de inauguración de la Universidad se celebró el 12 de octubre en el Teatro Degollado, considerado ya desde entonces el máximo recinto de la ciudad y fue presidida por el secretario de Educación Pública federal, José Manuel Puig Casauranc, y el gobernador Guadalupe Zuno; fueron universidades madrinas las de Salamanca, México, París y California, las cuales enviaron a sus representantes.

\section{CONCLUSIÓN}

El embeleso por un mesianismo que viene de la epopeya revolucionaria mexicana y concretizado en el campo virgen de la educación superior por Zuno en 1825, explica su vehemencia por el modelo estatal del laicismo universitario. ¿Qué más pedir? Si es el propio Estado quien provee a la institución de una institución humanista y plural. El discurso pronunciado por Zuno en $1957,{ }^{57}$ treinta

56 Como la Federación de Profesores Universitarios y asignaron ahí a un cacique del grupo universidad: Genaro Cornejo Cornejo y luego años después, con la influencia del surgimiento del sindicalismo universitario en la UNAM, los sindicatos de docentes (STAUdG) y de administrativos (STUdG).

57 José Guadalupe Zuno, “Discurso”, op. cit., (cita 50). 
y dos años después de la fundación que él mismo gestó muestra palmariamente, párrafo a párrafo que ese modelo bastaba para la conformación integral de su paradigma. Modelo que se sustentaba en la verticalidad y no en la colegiación, o en una colegiación muy dirigida, pues todos los puestos del colegio universitario, el Consejo, máximo órgano de dirección, eran atribución según la ley orgánica del Rector de la universidad, así que la democracia tantas veces agitada por las banderas estudiantiles en la localidad e incluso en Latinoamérica, no hacía falta. El poder universitario, -se desprende de su discurso- lo tenía todo.

El gobierno universitario estaba representado por el consejo Universitario, al igual que muchas universidades del mundo, pero la diferencia era que sus miembros eran los directores de Escuelas y Facultades, los cuales no eran elegidos, sino nombrados por el Rector, y esta a su vez, era nombrado por el gobernador, es decir por el propio Zuno. El Rector nombraba a los profesores, conjuntamente con los directores y hasta los empleados. ${ }^{58}$ Era entonces el jefe nato de la Universidad; ante esta situación ¿qué falta hacía la autonomía y la democracia?

Se pudiera pecar de presentismo al suponer que la democracia universitaria no estaba en los paradigmas de la época, pero no es así. En todos los congresos estudiantiles mexicanos y en los reclamos de la agitación mundial está el problema de la democracia. - de ahí que, ante los oídos sordos de los viejos regímenes heredados del siglo XIX, se escenificaron las revoluciones sociales del nuevo siglo. México tenía ya la suya, ahora había que construir las instituciones democráticas en todo el tejido social. La universidad era una arena propicia para contribuir a esa aspiración, pero Zuno de reservó ese derecho, y no solo él, podríamos decir que el naciente Estado mexicano ejerció una libertad "verticalizada", es decir concedida desde arriba.

Así fue en la Universidad de Guadalajara. En la Universidad nacional, fundada unos años antes y reformada en 1929 ante los reclamos de la gran protesta estudiantil, implantó su autonomía bajo un régimen de libertad compartida, plural. ${ }^{59}$ La de Guadalajara no, y eso sentó las bases para la cancelación histórica de libertad política en su campus. Hasta la fecha.

\section{FUENTES}

Archivo Histórico de Porfirio Díaz, (AHPD)

Archivo Histórico de la Universidad de Guadalajara, (AHUDG)

Ley Orgánica de la Universidad de Guadalajara. (LOUG). Ley del 25 se septiembre de 1925, decreto 2721.

58 Ley Orgánica de la Universidad de Guadalajara. (LOUG). Ley del 25 se septiembre de 1925, Decreto 2721.

59 La representación estudiantil en el consejo Universitario en 1929 de la Universidad Nacional ya con su autonomía, estará conformada por "dos alumnos inscritos como numerarios por cada facultad o escuela, un alumno y una alumna delegados de la federación estudiantil, electos, respectivamente por los alumnos y alumnas de la federación y un delegado designado por cada una de las asociaciones de exalumnos graduados. Reglamento interior del Consejo Universitario de la Universidad Autónoma de México, 1929, en Compilación de Legislación universitaria de 1910 a 1976. Comisión Técnica de Estudios legislativos. Tomo I. (México: UNAM, 1977), 18. 


\section{REFERENCIAS}

Aldana Rendón, Mario. "Enrique Díaz de León y su circunstancia política". En Enrique Díaz León: Filosofía y Acción Política, editado por Mario Aldana Rendón y Miguel Romero Morett. Guadalajara: Universidad de Guadalajara, 1988, 11-55.

Aldana Rendón, Mario. Manuel M. Diéguez y la revolución mexicana. Jalisco: El Colegio de Jalisco, 2006.

Ayon Zester, Francisco. Universidad de Guadalajara. Guadalajara: Instituto Jalisciense de Antropología e Historia, 1986.

Brindis, Schulamit Goldsmit. "Jalisco", en Contento y descontento en Jalisco, Michoacán y Morelos 19081911, editado por Graciela de Garay y otros. México, UIA, 1991, 27.

Camarena, Enrique Francisco. "La primera huelga de estudiantes universitarios". En Narraciones Tapatías, De Guadalajara Jal., De 1900 a 1958 (Guadalajara: Librería el estudiante, s.f), 237-242.

Castañeda, Carmen. La Educación en Guadalajara durante la Colonia. 1552-1821.México: CIESAS, 2012.

Córdoba, Arnaldo. "México. Revolución burguesa y política de masas". Cuadernos políticos, No. 13 (1977).

Cornejo, José Franco. Documentos referentes a la fundación, extinción y restablecimiento de la Universidad de Guadalajara. Guadalajara: Universidad de Guadalajara, 1972.

Cuño Bonito, Justo. "La universidad Latinoamericana en la encrucijada: amenazas, desafíos y soluciones". Revista Historia de la Educación Latinoamericana. Vol. 18 No. 26 (2016): 241- 277.

Díaz Tepepa, María Guadalupe. “Técnica y Tradición. Etnografía de la Escuela Rural y de su Contexto Familiar y Comunitario Investigación Administrativa". Investigación y Postgrado, No. 83 (1999).

Ducoing, Patricia. La Pedagogía en la Universidad de México 1851-1954. Tomo 1. México: CESU. UNAM, 1990, 94-95.

Espeleta, Justa y Eduardo Weiss. "Las escuelas rurales en zonas de pobreza y sus maestros: tramas preexistentes y políticas innovadoras". Revista Mexicana de Investigación Educativa, No. 1, 1996.

García Diego, Javier. “Movimientos estudiantiles durante la revolución mexicana (Estudio de caso de la participación de un grupo de clase media urbana)". En Los estudiantes, trabajos de historia y sociología, editado por Renate Marsiske. México: CESU-UNAM, 1989, 144.

Gilly, Adolfo. “Los Dos Socialismos Mexicanos”. Revista Nexos (1986).

Gramy, Misael Gradilla. 1993. El juego del poder y del saber. Tesis doctoral. El Colegio de México. México.

Iguiniz, Juan B. La Antigua Universidad de Guadalajara. México: UNAM, 1959.

Loaeza, Soledad. "La rebelión de la Iglesia". Revista Nexos (Junio de 1984):11-17.

Mantilla Trolle, Marina y Armando Martínez Moya. “La Universidad de Guadalajara a la hora de su fundación". En Universidad de Guadalajara: más de dos siglos de historia, editado por Gloria Angélica Hernández Obledo. Guadalajara: Universidad de Guadalajara, 2013.

Martínez, Ignacio. Pintura Mural Siglo XX. Jalisco en el Arte. Guadalajara: Editorial de Planeación y Promoción, 1960

Marsiske, Renate. "La autonomía universitaria. Una visión histórica y latinoamericana". Perfiles Educativos XXXII, (2010): 9-26.

Marsiske, Renate. “El Movimiento Estudiantil de 1929 y la Autonomía de la Universidad Nacional de México", Revista de Educación Superior 11, No. 44, (1982).

Mendoza Cornejo, Alfredo. Historia de la Universidad de Guadalajara. 1696-1994, Vol. 2. Guadalajara: Edición del autor, 2005.

Mendoza Cornejo, Alfredo. Organizaciones y Movimientos Estudiantiles En Jalisco de 1900 a 1937. Guadalajara: Universidad de Guadalajara, 1989.

Zaragoza Raso, José Luis. Crónica de la Real y Literaria Universidad de Guadalajara. Guadalajara: Instituto Jalisciense de Antropología e Historia, 1963.

Ortoll, Servando. "Turbas anti yanquis en Guadalajara en vísperas de la revolución del diez" Boletín histórico de Jalisco 1, 2‥ época, No. 2 (Mayo -agosto 1983): 2-15.

Pacheco, Lourdes C. “Fuimos a sembrar cultura. Los maestros y la construcción de la escuela rural 
mexicana". Investigación y Postgrado 28, No.1 (2013).

Pezet, José Luis. La Universidad Española. Siglos XVIII y XIX. México: Editorial Taurus, 1974.

Ramírez Flores, José. La revolución maderista en Jalisco. Guadalajara: Universidad de Guadalajara, 1992.

Ruiz, Ramón Eduardo. La gran rebelión. 1905-1904. México: Editorial Era, 1982.

Salvador, Humberto. Prólogo a El movimiento artístico de México, por Salvador Navarro Aceves, 1520. Quito: Editorial ELAN, 1935.

Semo, Ilán. "El cardenismo revisado: la tercera vía y otras utopías inciertas". Revista Mexicana de Sociología 55, No. 2 (1993).

Soto Arango, Diana y Lafuente, María Isabel. Autonomía y modelos universitarios en América Latina. Tomo II Bogotá, León. Universidad de León, España, Asociación Colombiana de Universidades. ASCUN. Universidad Pedagógica y Tecnológica de Colombia, RUDECOLOMBIA, 2007.

Tamayo, Jaime. "Zunismo y movimientos sociales" y “El enfrentamiento Zuno-Calles" en La revolución en las regiones. Vol. 2. Guadalajara: Universidad de Guadalajara, 1986.

Torres Sánchez, Rafael. Jalisco y la Revolución mexicana. Estructura, Economía y Cambio Institucional. México: Universidad Autónoma de México, 2001.

Pachecho Calvo, Ciriaco. "El Primer congreso internacional de estudiantes celebrado en México en 1921". Revista Universidad de México, Tomo III, No. 14 (Diciembre de 1931): 187.

Valle, Rafael Heliodoro, Francisco Castillo Nájera y Alfonso Alarcón. Añoranzas del primer congreso de Estudiantes (1910) (México: Instituto de Historia de Nicaragua y Centroamérica, 1943), 118.

Velázquez Albo, María de Lourdes. “El imaginario de la Universidad Nacional de México en 1910" (Ponencia presentada en el X congreso Nacional de Investigación Educativa. Área 9, Historia e Historiografía de la Educación", Veracruz, 21 al 25 de septiembre, 2009.

Zuno Hernández, José Guadalupe. Anecdotario del centro bohemio. Guadalajara: Pedro Rodríguez Lomelí, 1964.

Zuno, José Guadalupe. Reminiscencias de una vida. México: El Diario, 1972.

Zuno Hernández, José Guadalupe. La historia de la revolución en el Estado de Jalisco. México: Instituto Nacional de Estudios Históricos de la Revolución Mexicana, 1964.

Zuno, José Guadalupe. Discurso inaugural de la Fundación de Guadalajara, AHUG, Fondo Universidad de Guadalajara en el XXXII Aniversario de la Fundación de la Universidad de Guadalajara, 16 de marzo de 2012.

Zuno, José Guadalupe. “Discurso pronunciado en el XXXIII aniversario de la fundación de la Universidad de Guadalajara". En Reminiscencias de una vida, editado por José Guadalupe Zuno (Guadalajara: Biblioteca de autores jaliscienses, 1958), 173-188.

Zuno, José Guadalupe. Prólogo a Biografía de Prohombres Liberales por Nombres y apellidos del autor del libro. Guadalajara: Nombre de la editorial, 1970, 8.

Apellidos, nombres del autor. "Palabras de Ramón Córdoba", Revista de la Universidad de Guadalajara, No. 13 (1966).

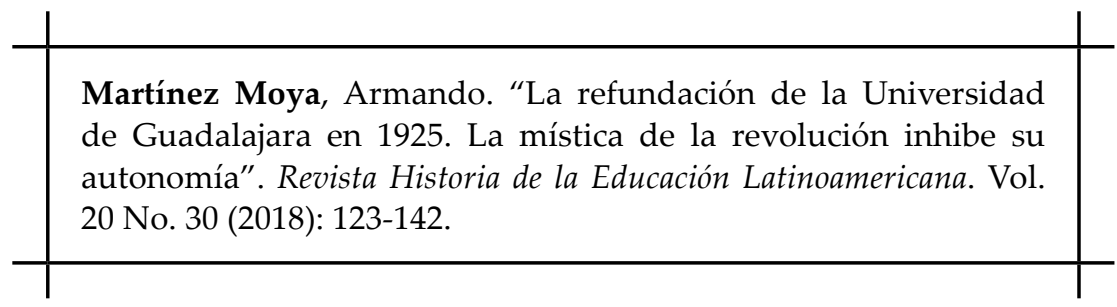

